\title{
Simulation of X-Ray Shielding Effect of Different Materials Based on MCNP5
}

\author{
Fanying Zhang, Xiaogang Zhao, Junxin Zhang \\ Applied Nuclear Technology in Geosciences Key Laboratory of Sichuan Province, Chengdu University of Technology, Chengdu, \\ China \\ Email: 1477591981@qq.com
}

How to cite this paper: Zhang, F.Y., Zhao, X.G. and Zhang, J.X. (2020) Simulation of X-Ray Shielding Effect of Different Materials Based on MCNP5. Open Access Library Journal, 7: e6727.

https://doi.org/10.4236/oalib.1106727

Received: August 19, 2020

Accepted: September 8, 2020

Published: September 11, 2020

Copyright () 2020 by author(s) and Open Access Library Inc.

This work is licensed under the Creative Commons Attribution International License (CC BY 4.0).

http://creativecommons.org/licenses/by/4.0/

\begin{abstract}
This article uses the Monte Carlo method and MCNP5 software to first simulate the X-ray energy spectrum of the tungsten target and the silver target. On this basis, using lead, tungsten and tungsten alloys (90\% tungsten, $7.1 \%$ nickel, and iron $2.9 \%$ ) as an X-ray shielding material, the shielding efficiency of these three materials at different thicknesses is calculated, and the results show that tungsten and tungsten alloy have better shielding effect than lead. For the X-rays of different energies generated by the tungsten target and the silver target, in order to achieve the same shielding effect, the X-rays generated by the tungsten target require a thicker shielding material.
\end{abstract}

\section{Subject Areas}

Nuclear Physics

\section{Keywords}

X-Ray Shielding, Tungsten Alloy, Monte Carlo Simulation

\section{Introduction}

X-rays can be divided into bremsstrahlung and characteristic X-rays. When high-speed moving electrons collide with objects, energy conversion occurs. The movement of electrons is blocked and loses kinetic energy. Part of the energy is converted into X-rays, and the other part is converted into the heat energy increases the temperature of the object [1]. The X-ray generator simulated in this article is based on this principle. The shielding of X-rays usually uses substances with higher atomic number and higher density, and lead is often used as a shielding material to absorb X-rays. The advantage of lead is its low price and high attenuation coefficient for $\mathrm{X}$ photons and gamma photons; but lead has low 
hardness, poor impact resistance, high temperature resistance and toxicity, and when the energy of X-ray photons is $40-80 \mathrm{keV}$ in between, the absorption capacity of lead to them is very weak, that is, the "weak absorption zone" of lead [2]. These characteristics make its application in certain occasions restricted. In contrast, as a high-density, high atomic number and non-toxic material, tungsten also has a good attenuation coefficient. Alloys with tungsten as the matrix and other elements, such as tungsten-nickel-iron alloys, can improve the overall properties of pure tungsten, such as strength and workability. Therefore, in practical applications, they are often used in the form of tungsten alloys [3]. In tungsten alloys, the mass content of tungsten is generally $85 \%$ to $99 \%$, and elements such as $\mathrm{Ni}, \mathrm{Cu}, \mathrm{Co}, \mathrm{Mo}$, and $\mathrm{Cr}$ are added to form high specific gravity alloys, which can be divided into W-Ni-Fe, W-Ni-Cu, W-Co, W-Ag and other series.

Monte Carlo method, also known as random sampling or statistical experiment method, is mainly used to simulate some random systems that cannot be generated by numerical values. At present, Monte Carlo methods and software are widely used in nuclear physics, medicine, materials science, system reliability and other fields at home and abroad, solving many problems that cannot be solved by traditional numerical simulation methods [4]. Commonly used Monte Carlo simulation software includes MCNP5, GEANT4, FLUKE, EGS [5], the MCNP5 used in this article. Monte Carlo simulation software MCNP5 can be used to simulate the transmission process of various particles such as protons, photons, electrons [6]. Based on MCNP5 simulation software, this paper simulates the X-ray energy spectrum of tungsten target and silver target. It mainly discusses the shielding effect of tungsten X-ray and silver X-ray when lead, tungsten, and tungsten nickel-iron alloy (tungsten 90\%, nickel 7.1\%, iron 2.9\%) have different thicknesses.

\section{MC Simulation of X-Ray}

Select silver and tungsten as the target materials respectively, and select an appropriate target thickness when the emitted electron energy is $100 \mathrm{keV}$, and the $\mathrm{X}$-ray energy spectra of silver and tungsten are simulated. The simplified MCNP5 model is shown in Figure 1.

The different thickness of the target material affects the count of the low-energy spectrum and the high-energy spectrum. As the target thickness increases, the count of high-energy bands increases first and then decreases. The

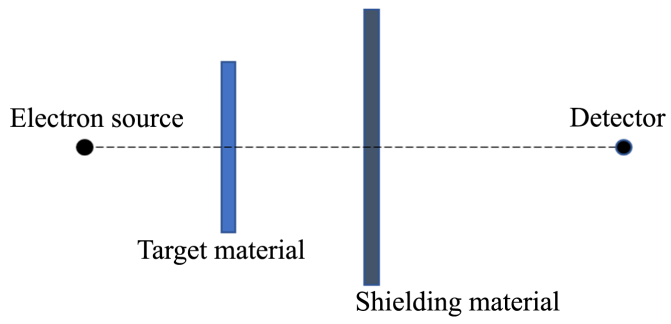

Figure 1. MCNP5 model of X-ray shielding. 
count of the low-energy spectrum decreases with the increase of the target thickness [7]. This is because when the thickness of the target material is thinner, the probability of low-energy photons generated by Compton scattering through the target material increases, resulting in a higher peak at the low-energy end of the X-ray spectrum. When a certain thickness is reached, due to the thickness of the shielding material much greater than the mean free path of low-energy photons, most of these low-energy Compton scattered photons are absorbed.

When the K-layer electrons are excited to produce an electron vacancy, the electrons in the shells of higher energy levels such as L, M, and $\mathrm{N}$ may fill this electron vacancy. The $\mathrm{X}$-rays emitted by filling the electron vacancies in the $\mathrm{K}$ shell are called $\mathrm{K}$ characteristic X-rays, and the X-rays emitted by filling the electron vacancies in the $\mathrm{L}$ shell are called $\mathrm{L}$ characteristic X-rays. The characteristic X-rays emitted when the electrons in the L layer and the $\mathrm{M}$ layer are filled into the $\mathrm{K}$ layer are represented by $K_{\alpha}$ and $K_{\beta}$ [8]. Taking into account the small energy difference between the sub-levels of electrons in the same orbit, the characteristic X-rays emitted when electrons of different sub-levels on the $\mathrm{L}$ layer are filled into the $\mathrm{K}$ layer can be divided into $K_{\alpha 1}, K_{\alpha 2}$. The characteristic $\mathrm{X}$-rays emitted when electrons of different sub-energy levels on the M layer are filled into the $\mathrm{K}$ layer can be divided into $K_{\beta 1}$ and $K_{\beta 2}$. In the same way, there are $L_{\alpha 1}, L_{\alpha 2}, L_{\beta 1}$, and $L_{\beta 2}$ for the characteristic X-rays of the L series. [9] [10].

The X-ray spectrum consists of a bremsstrahlung spectrum and a characteristic X-ray spectrum. The characteristic X-ray spectrum is superimposed on the continuous bremsstrahlung spectrum [11]. The thickness of the tungsten target is selected as $0.1 \mathrm{~mm}$ in this paper, and its X-ray spectrum is shown in Figure 2. The X-ray spectrum of tungsten mainly has 5 characteristic X-ray peaks, the leftmost energy is $8.9 \mathrm{keV}$ is a characteristic peak of L series; the peak is higher at energy $59.3 \mathrm{keV}, 58.0 \mathrm{keV}$, here are the characteristic X-ray peaks $K_{\alpha 1}$ and $K_{\alpha 2}$ of the K series. And at the energies of $67.2 \mathrm{keV}$ and $69.1 \mathrm{keV}$ are the characteristic X-ray peaks $K_{\beta 1}$ and $K_{\beta 2}$ of the $\mathrm{K}$ series. The thickness of the $\mathrm{Ag}$ target is selected to be $0.002 \mathrm{~mm}$, and its X-ray spectrum is shown in Figure 3.

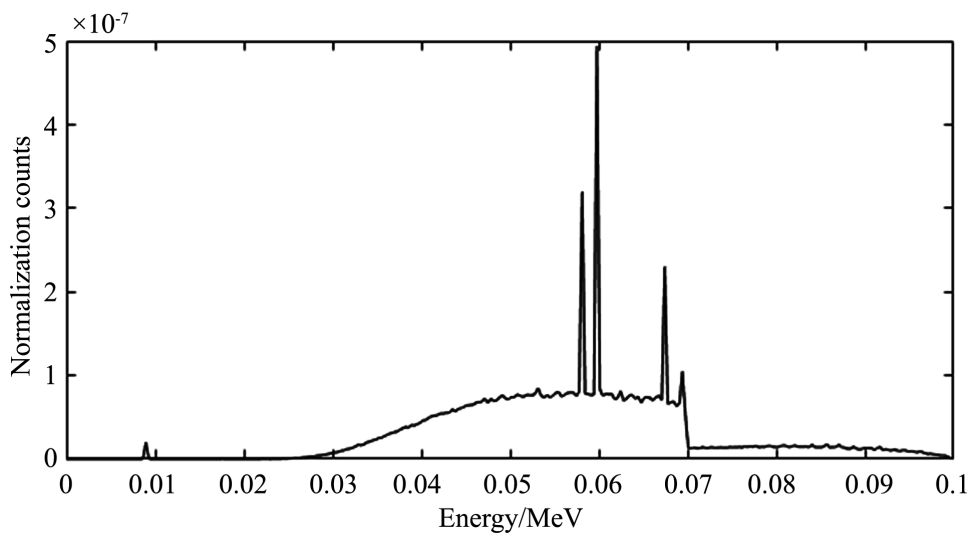

Figure 2. X-ray spectrum of tungsten target. 


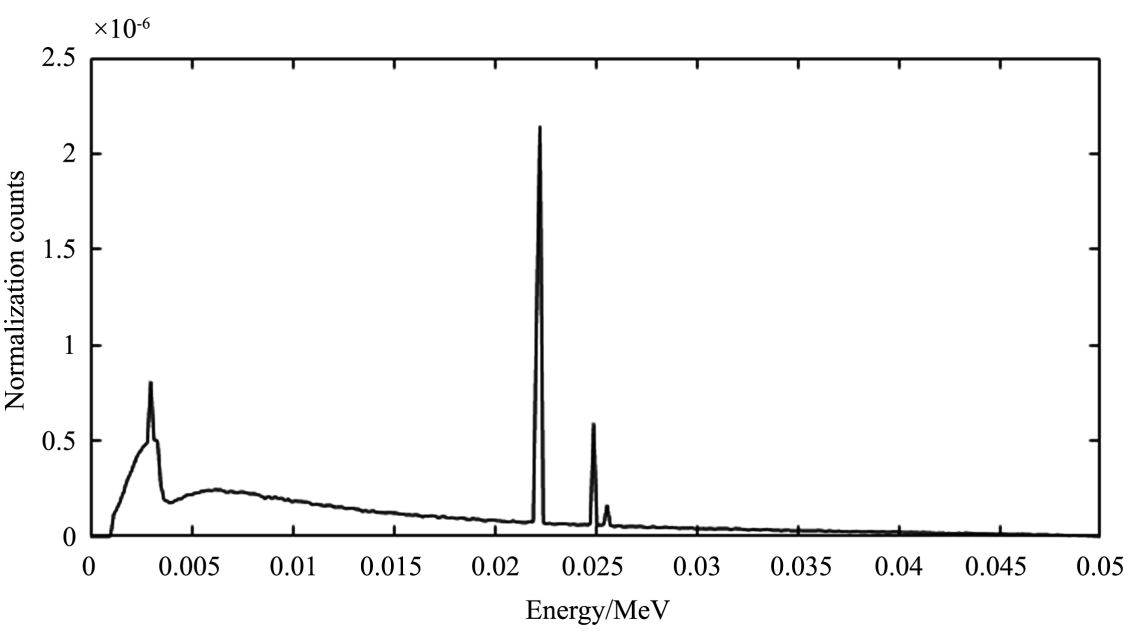

Figure 3. X-ray spectrum of silver target.

There is a characteristic X-ray peak of the $\mathrm{L}$ series at $3 \mathrm{keV}$. The two characteristic X-ray peaks of the $\mathrm{K}$ series, $K_{\alpha 1}$ and $K_{\alpha 2}$ correspond to the energies $E_{K_{\alpha 1}}=22.2 \mathrm{keV}$ and $E_{K_{\beta 1}}=24.9 \mathrm{keV}$, which represent the characteristic energy values of the $\mathrm{K}$ series characteristic $\mathrm{X}$-rays of the silver element.

\section{Research on the Shielding Performance of Lead, Tungsten and Tungsten Alloy}

Shielding materials of different thicknesses were added behind the target to simulate the effects of lead, tungsten, and tungsten-nickel-iron alloy (90\% tungsten, $7.1 \%$ nickel, and $2.9 \%$ iron) on the X-rays generated by the tungsten and silver targets Shielding effect. For the simulation results, the shielding rate is used to compare the shielding effect [12]. The count before the shielding material is $I_{0}$ and the count after the shielding material is $I$. The expression for measuring its filtering ability is: $T=\left(I_{0}-I\right) / I_{0}$.

For three different materials, the shielding effect when the material thickness is $0.1 \mathrm{~mm}$ to $0.8 \mathrm{~mm}$ is simulated. From Table 1 , the following conclusions can be drawn: 1) the X-ray shielding rate of these three materials varies with the material As the thickness increases, 2) the X-ray shielding rate of tungsten and tungsten alloy is always higher than that of $\mathrm{Pb}, 3$ ) for tungsten and tungsten alloy, the shielding rate of tungsten is between $0.1 \mathrm{~mm}$ and $0.6 \mathrm{~mm}$. Higher than the shielding rate of tungsten alloy, at $0.7 \mathrm{~mm}$ and $0.8 \mathrm{~mm}$, the shielding rate of tungsten alloy is slightly higher than that of tungsten.

\section{Analysis of Simulation Results}

The attenuation of the X-ray intensity is related to the attenuation coefficient of the absorbing material. The material with a large attenuation coefficient attenuates X-ray photons more than the material with a low attenuation coefficient [13]. In the range of $0-100 \mathrm{keV}$, the mass attenuation coefficient of lead is larger than that of tungsten. However, due to the higher density of tungsten, in general, 
Table 1. The shielding effect of lead, tungsten and tungsten-nickel-iron alloy on tungsten $\mathrm{X}$-rays and silver X-rays.

\begin{tabular}{ccccccc}
\hline \multirow{2}{*}{ Thickness/mm } & \multicolumn{3}{c}{ X-ray shielding rate of $\mathrm{W}$} & \multicolumn{3}{c}{ X-ray shielding rate of Ag } \\
\cline { 2 - 7 } & $\mathrm{Pb}$ & $\mathrm{W}$ & $\mathrm{W}-\mathrm{Ni}-\mathrm{Fe}$ & $\mathrm{Pb}$ & $\mathrm{W}$ & $\mathrm{W}-\mathrm{Ni}-\mathrm{Fe}$ \\
\hline 0.1 & 0.7561 & 0.8124 & 0.7964 & 0.9568 & 0.9733 & 0.9690 \\
0.2 & 0.8801 & 0.9182 & 0.9073 & 0.9795 & 0.9903 & 0.9877 \\
0.3 & 0.9289 & 0.9621 & 0.9496 & 0.9885 & 0.9955 & 0.9946 \\
0.4 & 0.9492 & 0.9760 & 0.9617 & 0.9927 & 0.9982 & 0.9968 \\
0.5 & 0.9614 & 0.9860 & 0.9798 & 0.9946 & 0.9990 & 0.9983 \\
0.6 & 0.9756 & 0.9940 & 0.9879 & 0.9965 & 0.9994 & 0.9993 \\
0.7 & 0.9919 & 0.9940 & 0.9960 & 0.9967 & 0.9995 & 0.9996 \\
0.8 & 0.9939 & 0.9960 & 0.9980 & 0.9984 & 0.9996 & 0.9996 \\
\hline
\end{tabular}

the minimum density of tungsten nickel-iron alloy can also reach $16.85 \mathrm{~g} / \mathrm{cm}^{3}$. Therefore, the linear attenuation coefficient of tungsten is the range of $0-100$ $\mathrm{keV}$ is almost all larger than lead. For tungsten and lead of the same thickness, the radiation shielding performance is definitely better than that of lead. For the same shielding effect, the shielding thickness is smaller when tungsten is used, that is, the shielding volume is the smallest, and the volume is larger when using lead.

Among the tungsten alloys selected in this paper, tungsten accounts for $90 \%$, nickel accounts for $7.1 \%$, and iron accounts for $2.9 \%$. Compared with the shielding effects of tungsten and tungsten nickel-iron alloys, the shielding efficiency of tungsten is only slightly higher than that of tungsten alloy. This shows that $90 \%$ of tungsten in the tungsten-nickel-iron alloy plays a leading role in the shielding effect, and the shielding effect of tungsten alloy mainly depends on the mass percentage of tungsten.

The energy of X-rays produced by tungsten targets and silver targets is different. Tungsten X-rays are counted more frequently from $40 \mathrm{keV}$ to $70 \mathrm{keV}$, and their $k_{\alpha 1}$ characteristic X-ray energy reaches $59.3 \mathrm{keV}$, while silver X-ray counts are mainly concentrated in from $2 \mathrm{keV}$ to $10 \mathrm{keV}$, the characteristic X-ray energy of $k_{\alpha 1}$ is only $22.2 \mathrm{keV}$. For these two different energy X-ray energies, in order to achieve the same shielding effect, a thicker shielding material is required for the X-rays generated by the tungsten target.

\section{Conclusions}

This article uses MCNP5 simulation to compare the X-ray shielding effects of lead, tungsten and tungsten-nickel-iron alloys on tungsten and silver. Combined with actual applications, the following factors need to be considered when ensuring the shielding efficiency: 1) When selecting a shielding material, in addition to the shielding effect of the material, the strength and workability of the material must also be considered. Tungsten alloy has both good comprehensive 
performance and excellent shielding effect, which is a good X-ray shielding material. 2) When shielding X-rays, you need to consider the X-ray energy to select suitable materials. Higher-energy X-rays require thicker materials to shield. For lower-energy X-rays, it is not necessary to choose strong shielding ability, price expensive tungsten alloys are used for shielding, and the appropriate thickness of lead can also be selected to achieve a shielding rate similar to that of tungsten alloys. For example, for the X-ray generated by the silver target in this article, 0.4 $\mathrm{mm}$ lead can also achieve a shielding effect similar to tungsten and tungsten alloys.

In the actual X-ray protection, the radiation protection performance of the material, the mechanical performance of the material, the compression performance, the environmental protection characteristics and the price of the material should be comprehensively considered to select the shielding material reasonably.

\section{Acknowledgements}

Thanks to Xiaogang Zhao for his suggestions on the selection of X-ray shielding materials in this article and Junxin Zhang for his opinions on the processing of the relevant data in the charts in this article.

\section{Conflicts of Interest}

The authors declare no conflicts of interest regarding the publication of this paper.

\section{References}

[1] Liao, L. and Qiu, X. (2010) Optimized Design of Shielding Material Component Content. Nuclear Electronics and Detection Technology, 30, 118-120.

[2] Zhu, Z. (2019) MC Simulation of Medical X-Rays and Optimization Design of Protective Materials. Nanjing University of Aeronautics and Astronautics, Nanjing.

[3] Wang, J. and Zou, S. (2011) Comparative Study on the Performance of Tungsten and Lead as $\gamma$-Ray Shielding Materials. Journal of Nanhua University, 25, 19-22.

[4] Du, C. and Xu, X. (2009) Monte Carlo Calculation of X-Ray Transmission Spectrum. The Second National Symposium on Nuclear Technology and Application Research, Mianyang, 1 May 2009, 599-602.

[5] Gu, R. (2016) EDXRF Analysis of the Best Detection Device for Heavy Metal Cd in Rice. Chengdu University of Technology, Chengdu.

[6] Xu, S. (2010) Application of Monte Carlo Method in Experimental Nuclear Physics. Atomic Energy Press, Beijing.

[7] Zhang, Q., Ge, L. and Yi, G. (2013) MC Simulation Analysis of the Influence of Transmission Type Micro X-Ray Tube Target Thickness on the Output Spectrum. Spectroscopy and Spectral Analysis, 33, 2232-2234.

[8] Qian, Y. (2009) Application of Monte Carlo Method in EDXRF Analysis. Chengdu University of Technology, Chengdu.

[9] Ji, A., Tao, G. and Zhuo, S. (2003) X-Ray Fluorescence Spectroscopy Analysis. Science Press, Beijing. 
[10] Cao, L., Ding, Y. and Huang, Z. (1998) Energy Dispersive X-Ray Fluorescence Method. Chengdu University of Science and Technology Press, Chengdu.

[11] Yang, Q., Ge, L. and. Gu, Y. (2013) Theoretical Calculation of Target Thickness for Miniature X-Ray Tube and Simulation of Output Spectrum. Spectroscopy and Spectral Analysis, 4, 1130-1134.

[12] Zhu, Z., Luo, W. and Chai, F. (2019) MC Simulation of Medical Diagnostic X-Ray Field and Optimization Design of Composite Shielding Materials. Journal of East China University of Technology (Natural Science Edition), No. 2, 169-172.

[13] Chen, X., Wei, C. and Sun, J. (2019) Simulation Analysis and Experimental Verification of X-Ray Shielding Performance of Tungsten Alloy. Chinese Stereology and Image Analysis, 24, 9-15. 